The Low Surface Brightness Universe, IAU Col. 171

ASP Conference Series, Vol. 170, 1999

J. I. Davies, C. Impey and S. Phillipps, eds.

\title{
What We Don't Know About the Universe
}

\author{
Chris Impey
}

Steward Observatory, University of Arizona, Tucson, AZ 85721, USA

\begin{abstract}
.
Despite progress on many fronts in cosmology, outstanding questions remain. What is the nature of the dark matter? Is the inflationary big bang model viable? Must we accept a non-zero cosmological constant? Do we know the true population of galaxies? What is the range of star formation histories in the universe? Can gravity alone explain the large scale structure we observe? Studies of the low surface brightness universe may provide the answers to many of these questions.
\end{abstract}

\section{Introduction}

It was the best of times, it was the worst of times. In many ways, this is a golden age in cosmology. Large new telescopes and efficient detectors have enabled surveys of unprecedented depth and scope. There are nearly a hundred thousand published galaxy redshifts; soon there will be several million. Quasars and other AGN are being successfully used to measure diffuse baryons (via absorption lines) and the distribution of dark matter (via gravitational lensing) over $90 \%$ of the lookback time. There is good evidence that our universe is adequately described by the hot big bang model - with understandable uncertainty over the first billion years or so when gravitational collapse was nonlinear and many stellar systems formed (e.g. Peebles 1993). Supercomputer models successfully reproduce the observed basic characteristics of large scale structure.

On the other hand, we remain ignorant of important aspects of cosmology (for an overview, see Bahcall and Ostriker 1997). The need for dark matter is inescapable, but the most viable candidates for a cold dark matter particle involve new or unknown physics. We believe that gravity can explain the large scale motions and clustering of galaxies, but neither supercomputer simulations nor pure theory can account for all of the existing observations. The quest to measure the parameters of the standard model continues. This was the goal of the Palomar 200 inch telescope when it was built in the 1930s, it was a goal of the Hubble Space Telescope when it was launched nearly ten years ago, and it is a goal of current and prospective 8-10 meter telescopes and microwave satellites. Only those who fall prey to the ancient Greek sin of hubris would claim that the end of cosmology is in sight. Astronomy is an observational science, and the universe has shown an impressive ability to surprise us. 


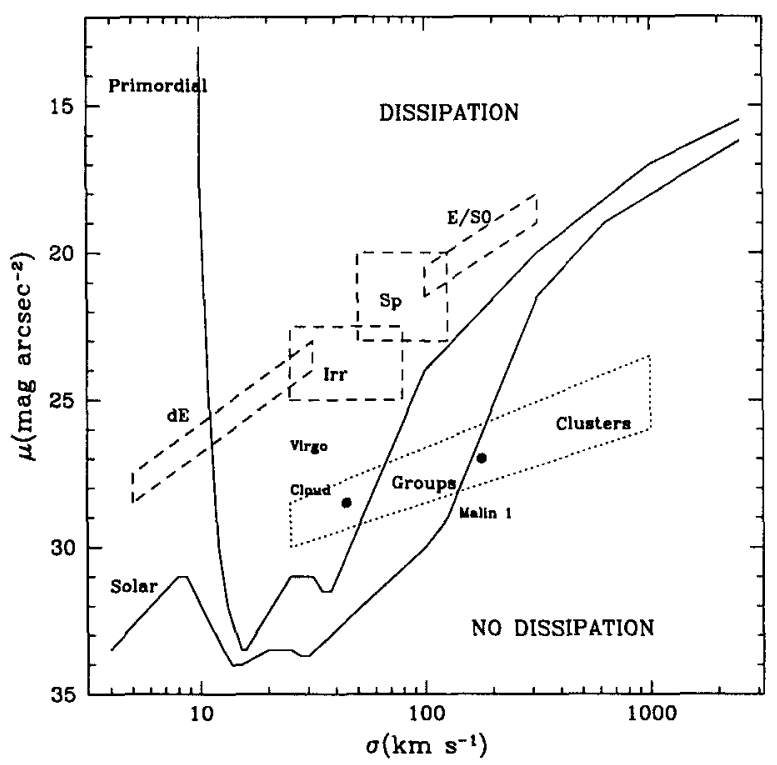

Figure 1. Collapsed structures in the universe, assuming dissipation by intercloud collisions, after Efstathiou \& Rees (1983).

\section{What We Don't Know About Stars}

The "bread and butter" of the universe is the conversion of gas into stars. We know a lot about the modes of star formation in local galaxies all along the Hubble sequence (Kennicutt 1998). We are also getting a basic idea from deep surveys of the history of star formation in the universe (Madau, Pozzetti, \& Dickinson 1998). However, it is also clear that there are dim and unrecognized repositories of baryons. At low redshift, the serendipitous discovery of Malin 1 - still that largest and most gas-rich galaxy known - is a reminder that star formation in disks can be slow and very inefficient (Impey \& Bothun 1989). Figure 1 shows that the lowest surface brightness galaxies have properties quite distinct from normal stellar systems on the Hubble sequence. At high redshifts, galaxies can escape detection due to the severe effects of surface brightness dimming or due to dust obscuration. We still do not know the full range of star formation histories in the universe. The luminosity-weighted integral $\int \phi(L) L d L$ appears to converge not far below $L_{*}$, but there are indications of a steepening fainter than $M_{B}=-16$ (see the discussion in Impey \& Bothun 1997). The most abundant stellar system in the universe is a gas-rich dwarf galaxy, examples of which have rarely been studied outside the Local Supercluster. The motivation to look for additional baryons is strong; $\Omega_{\text {lum }}$ from the integral of the galaxy luminosity function is a factor of 2-3 below the lower bound on $\Omega_{\text {baryon }}$ from nucleosynthesis arguments (Copi, Schramm, \& Turner 1995; Persic \& Salucci 1992; Bristow \& Phillipps 1994). 


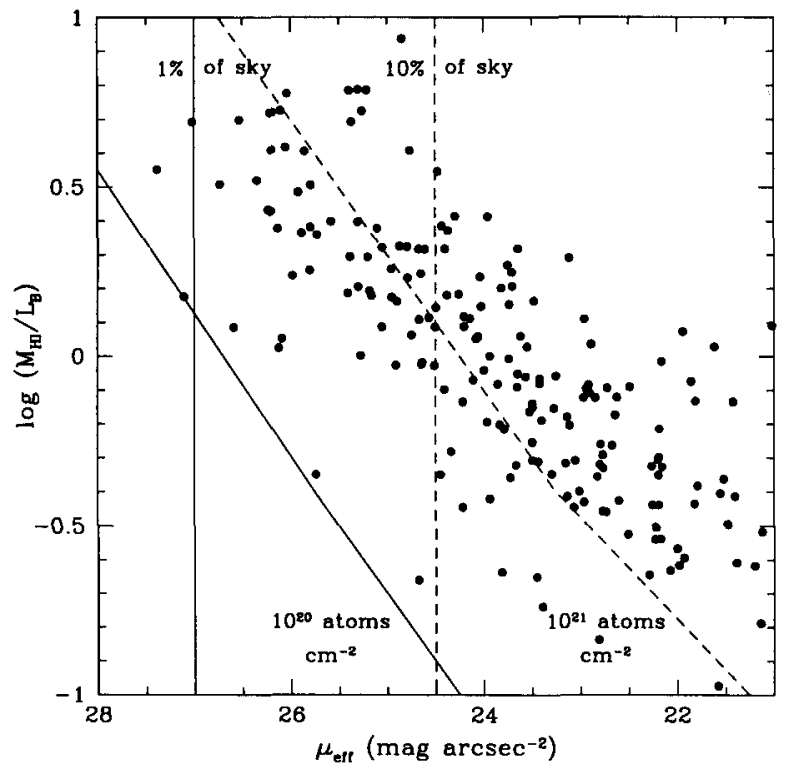

Figure 2. The gas mass fraction of LSB galaxies from the APM survey increases with decreasing surface brightness. There are selection effects against finding objects that are diffuse in either gas or stars.

\section{What We Don't Know About Gas}

Astronomers typically use galaxies as markers of space in many of the standard cosmological tests. Galaxies are easy to detect. Notably, the upcoming Sloan Digital Sky survey will produce digital information for about 100 million of them. All optical surveys will undercount galaxies that are diffuse or small or unevolved (in the sense of having converted a small fraction of the gas mass into stars). While cold gas can be detected effectively by the $21 \mathrm{~cm}$ line of neutral hydrogen, the limited bandwidth and sensitivity of "blind" radio surveys mean that they probe relatively small volumes (Schneider, Spitzak, \& Rosenberg 1998). Figure 2 shows that galaxies from the APM survey (Impey et al. 1996) have a strong trend of increasing gas richness with lower surface brightness (McGaugh \& de Blok 1997). In this diagram, there is strong surface brightness selection galaxies fainter than $10 \%$ of the sky level are under-represented and galaxies fainter than $1 \%$ of the sky level do not make it into most optical catalogs. The high surface brightness galaxies have have HI column densities of a few times $10^{21}$ atoms $\mathrm{cm}^{-2}$ and the low surface brightness galaxies have $\mathrm{HI}$ columns ten times lower. At any particular surface brightness, radio surveys only detect the most gas-rich galaxies. Below a few times $10^{19}$ atoms $\mathrm{cm}^{-2}$, disk star formation is inhibited (Kennicutt 1989), and we know very little about the universe at these low column densities. 


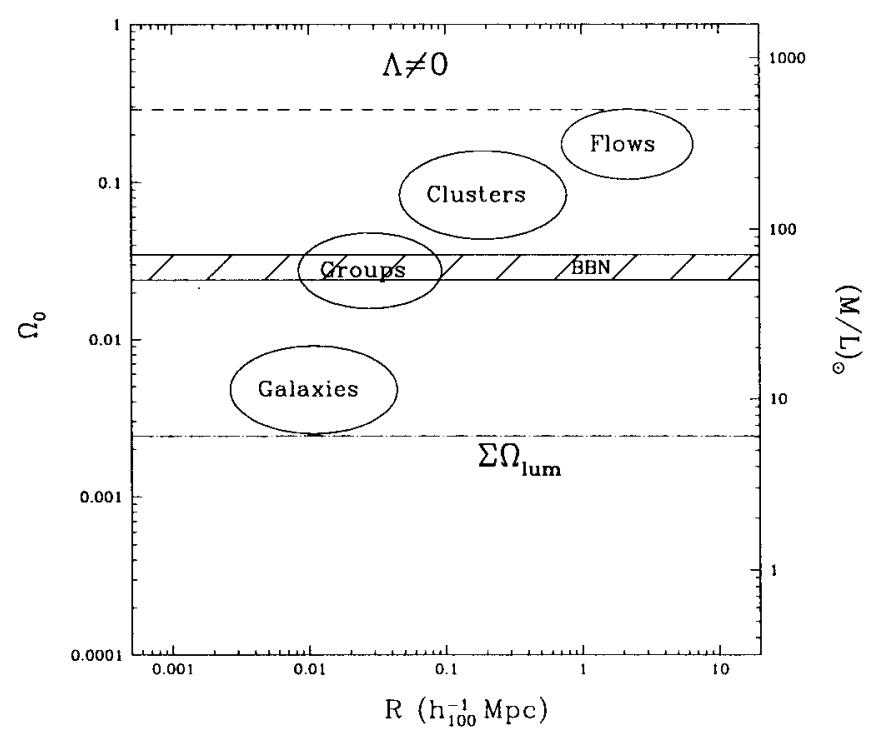

Figure 3. The density of matter on different scales. The dot-dashed line indicates the integral over luminous matter in galaxies and the striped region shows the baryon bounds from big bang nucleosynthesis.

\section{What We Don't Know About Dark Matter}

The nature of dark matter is the critical unresolved issue in cosmology. On the scale of individual galaxies, the evidence for dark matter is incontrovertible (for a review, see Ashman 1992). From kinematic studies on cosmological scale, two conclusions emerge, as illustrated by Figure 3. Clusters show dark matter in excess of the plausible upper bound from nucleosynthesis, indicating that most of the dark matter is nonbaryonic (Bahcall, Lubin, \& Dorman 1995). Structure formation theories would require this dark matter to be non-relativistic at the epoch of recombination, or "cold." This in turns implies a particle physics solution to a cosmology problem, with no experimental data to guide us at this time. Second, cluster evolution and mass-to-light ratios point to a value of $\Omega_{0}$ well below one (setting aside for a moment the ambiguous results from large scale bulk flows). If this is true, then the spatially flat universe that is a natural consequence of the inflationary big bang model can only be recovered with the addition of a non-zero cosmological constant. Unfortunately, we have no theoretical expectation for why $\Lambda$ should have a cosmologically interesting value. Few people are willing to contemplate a deeper level of ignorance concerning our theory of gravity, yet there are puzzling problems in the dynamics of galaxies that deserve continued attention (Milgrom 1989; McGaugh \& de Blok 1998). 


\section{Bright Icebergs}

Following earlier speculation by Zwicky (1957), Arp (1965), and Peebles (1971), Disney (1976) proposed the existence of galaxies that lurked mostly below the level of the sky brightness. The distribution of surface brightness shows no sign of a turnover down to the limit of current surveys (McGaugh 1996; Dalcanton et al. 1997). This raises the issues of whether there is a limit to the diffuseness of a stellar system and what the mode of star formation is in a disk with only a few $\mathrm{M}_{\odot} \mathrm{pc}^{-2}$. Even if Malin-type disks do not contribute much to the luminosityweighted integral of galaxies, they are important laboratories for studying the distribution of dark matter in large halos (Navarro, Frenk \& White 1997). Also, the large size of the gas disks can result in a significant cross section to quasar absorption, perhaps accounting for some fraction of the damped Lyman- $\alpha$ systems.

\section{Dim Icebergs}

Supercomputer simulations that incorporate hydrodynamics have been able to reproduce many of the large scale features of the universe. Cold dark matter modulates the formation of a filamentary web of low column density gas (e.g. Ostriker \& Cen 1996). Quasar absorption can be used to trace the distribution and metallicity of this gas over nearly ten orders of magnitude in column density. Figure 4 shows the different scales probed by hydrogen absorbers of different column densities. The highest column densities correspond to damped Lyman- $\alpha$ lines due to galaxy disks or their progenitors at high redshift. More modest column densities trace metal-enriched gas in the extended halos of bright galaxies. At $z \sim 2$ most of the baryons in the universe are in diffuse structures of about $10^{14}$ atoms $\mathrm{cm}^{-2}$ (Hernquist et al. 1996; Miralda-Escude et al. 1996). Most of this gas has probably been heated to a high temperature by the present epoch (Cen \& Ostriker 1998). Galaxies are the whitecaps that float on this churning sea of diffuse baryons and dark matter.

\section{Dark Icebergs}

It is possible to find galaxies that are dark matter dominated across the size spectrum - from giant low surface brightness disks to gas-rich dwarfs. Perhaps there are dark halos where the baryonic component is negligible. There have been claims of "dark galaxies" based on the incidence of close quasar pairs where no lens is visible, even though a massive galaxy would be required to cause the observed image splitting (Hawkins 1997). Counter to this claim is the fact that radio and optical surveys do not show the same incidence of close quasars with no visible lensing galaxy (Kochanek, Falco, \& Munoz 1998). The alternative explanation is that the quasars are bound pairs, with interaction fuelling the activity in both AGN. At the other end of the mass scale, there is a clear expectation that dark halos exist, since the mass function in hierarchical clustering models is much steeper than the faint end of the galaxy luminosity function. Small halos may not be able to make or retain substantial numbers of stars. 


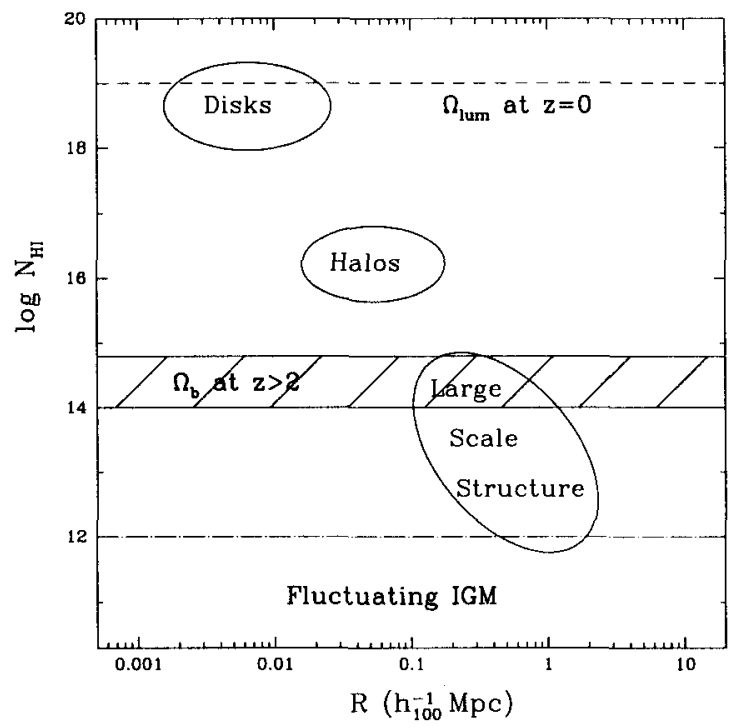

Figure 4. The column density and characteristic size of hydrogen quasar absorbers over ten decades in column density. The dot-dashed line is the level at which individual absorbers blend into a continuously fluctuating Gunn-Peterson effect. The dashed line is the mass density in luminous material measured locally.

\section{What We Don't Know About the Universe}

Cosmology has moved from its pioneering phase into its middle years, but the end game is not in sight yet. Figure 5 is a schematic view of the basic cosmological parameters, shown in terms of their level of uncertainty. The right hand side of the bar or arrow indicates the level of knowledge that we have now or can anticipate in the near future (for a recent overview, see Turok 1997). Only the Hubble constant is approaching a level of $10 \%$ precision, although there is still discordance between results from the conventional chain of of distance indicators and direct techniques (that depend on supernovae, gravitational lensing, and the Sunyaev-Zeldovich effect). Direct measurement of space curvature remains elusive, but the matter density is its proxy in the absence of substantial vacuum energy. The best evidence indicates that the universe cannot be closed by matter, whatever its form. After a series of adjustments to the stellar models, the derived ages of globular clusters have diminished to the point that they can accommodate a flat matter-dominated universe, provided that $H_{0}<67 \mathrm{~km} \mathrm{~s}^{-1}$ $\mathrm{Mpc}^{-1}$ (Chaboyer et al. 1998). The issue of the cosmological constant is still unresolved. Finally, we are gradually defining two key attributes of the universe after it begins to form structures - the star formation rate over a Hubble time and the power spectrum of density fluctuations.

The successful measurement of cosmological parameters is leaving us with some deep mysteries. If the cosmological constant can be ruled out, then we need a natural explanation for an open universe without sacrificing the virtues of the 


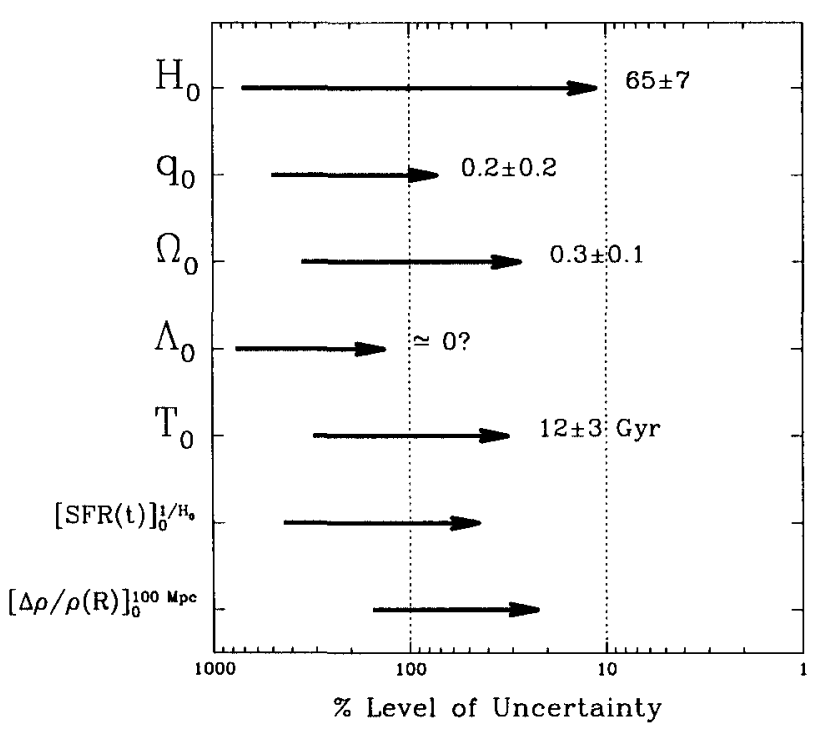

Figure 5. Current estimates of cosmological parameters. The end of the arrows indicate the precision of recent and prospective measurements. The diagram does not account for systematic errors that might affect each of these quantities.

inflationary big bang model. If standard inflation is saved with a vacuum energy term, we must explain why its contribution to the density parameter (based on unknown physics) is similar in magnitude to the matter density. Either way, we have to understand why baryons and dark matter particles - two fundamentally distinct microscopic phenomena - occur with roughly similar cosmic density. The invocation of anthropic arguments is merely a distraction as we try to refine our cosmological models. Ignorance can be a virtue; we should embrace it if it leads us to think of new and creative ways to understand the universe.

Acknowledgments. Thanks go to Mike Disney for illuminating much of our ignorance concerning galaxies, and for coming up with creative ways to counter it. Thanks to Jon Davies and the Local Organizing Committee for arranging a lively and convivial meeting. I acknowledge grant support from the National Science Foundation under AST-9003158 and AST-9617826, and from the International Programs Office of the University of Arizona.

\section{References}

Arp, H.C. 1965, ApJ, 145, 402

Ashman, K.M. 1992, PASP, 104, 1109

Bahcall, N.A., Lubin, L.M., \& Dorman, V. 1995, ApJ, 447, 81 
Bahcall, J.N. \& Ostriker, J.P. eds. 1997, Unsolved Problems in Astrophysics, Princton: Princeton University Press

Bristow, P.D., \& Phillipps, S. 1994, MNRAS, 267, 13

Cen, R., \& Ostriker, J.P. 1998, ApJ, in press

Chaboyer, B., Demarque, P., Kernan, P.J., \& Krause, L.M. 1998, ApJ, 494, 96

Copi, C.J., Schramm, D.N., \& Turner, M.S. 1995, Science, 267, 192

Dalcanton, J.J., Spergel, D.N., Gunn, J.E., Schmidt, M., \& Schneider, D.P. 1997, AJ, 114, 635

Disney, M.J. 1976, Nature, 263, 573

Hawkins, M.R.S. 1997, A\&A, 328, L25

Hernquist, L., Katz, N., Weinberg, D.H., and Miralda-Escude, J. 1996, ApJ, 457,51

Impey, C.D. \& Bothun, G.D. 1989, ApJ, 341, 89

Impey, C.D. \& Bothun, G.D. 1997, ARA\&A, 35, 267

Impey, C.D., Sprayberry, D., Irwin, M.J., \& Bothun, G.D. 1996, ApJS, 105, 209

Kennicutt, R.C. 1989 , ApJ, 344, 685

Kennicutt, R.C. 1998, ARA\&A, 36, 189

Kochanek, C.S., Falco, E.E., \& Munoz, J.A. 1998, ApJ, in press

Madau, P., Pozzetti, L., \& Dickinson, M. 1998, ApJ, 498, 10

McGaugh, S.S. 1996, MNRAS, 280, 337

McGaugh, S.S., \& de Blok, W.J.G. 1997, ApJ, 481, 689

McGaugh, S.S., \& de Blok, W.J.G. 1998, ApJ, 499, 41

Milgrom, M. 1989, ApJ, 338, 121

Miralda-Escude, J., Cen, R., Ostriker, J.P., \& Rauch, M. 1996, ApJ, 471, 582

Ostriker, J.P., \& Cen, R. 1996, ApJ, 464, 27

Navarro, J., Frenk, C.S., \& White, S.D.M. 1997, ApJ, 490, 493

Peebles, P.J.E. 1971, Physical Cosmology, Princeton: Princeton University Press

Peebles, P.J.E. 1993, Principles of Physical Cosmology, Princeton: Princeton University Press

Persic, M., \& Salucci, P. 1992, MNRAS, 258, 14P

Schneider, S.E., Spitzak, J.G., \& Rosenberg, J.L. 1998, ApJ, 507, L9

Turok, N. ed. 1997, Critical Dialogs in Cosmology, Singapore: World Scientific Publishing

Zwicky, F. 1957, Morphological Astronomy, New York: Springer Verlag 


\section{Searching for LSB - VIII}

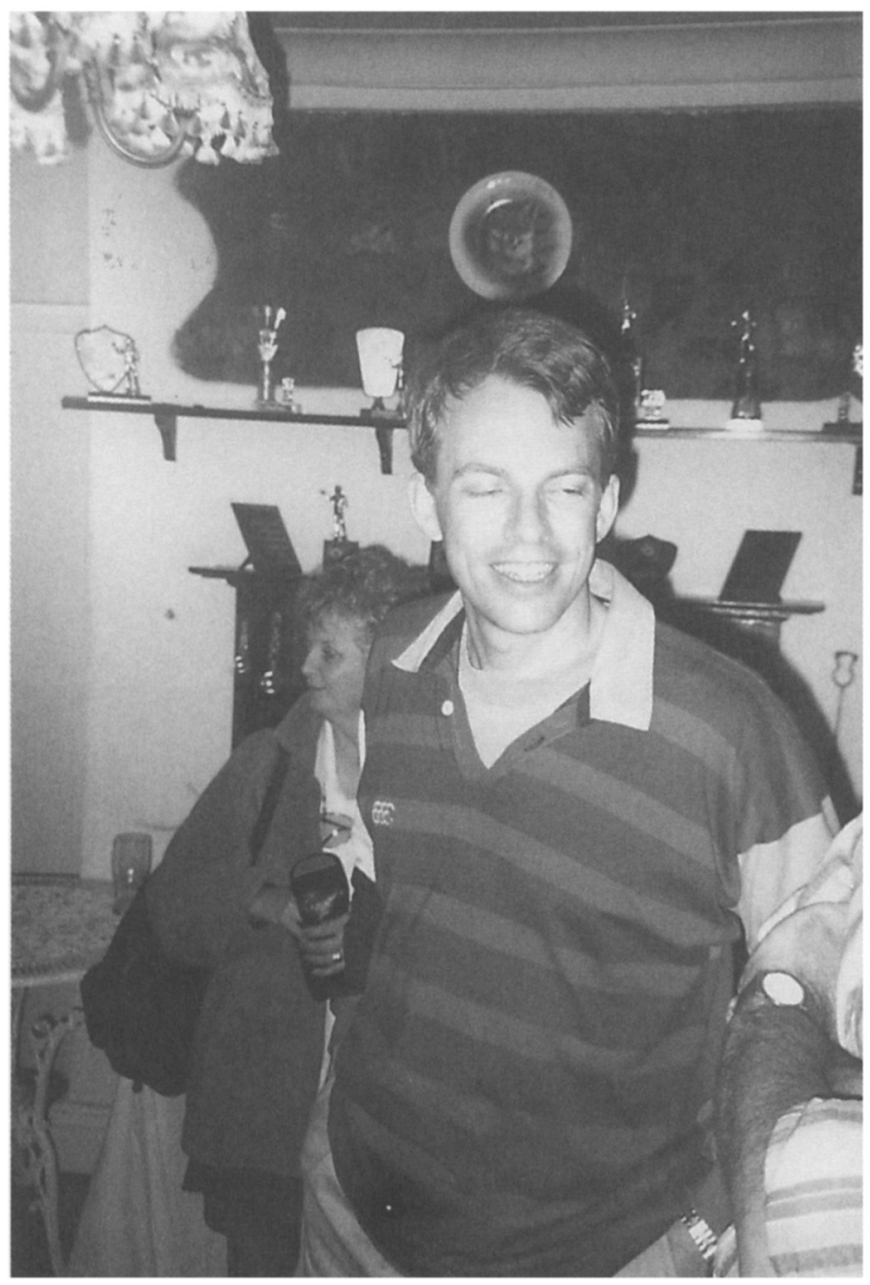

\section{At last, Large Supplies of Beer !}

